\section{RMD Open}

Rheumatic \& Musculoskeletal Diseases

\title{
Treatment of polyarteritis nodosa with tocilizumab: a new therapeutic approach?
}

\author{
Aurélie Saunier, ${ }^{1}$ Nahéma Issa, ${ }^{1}$ Marie-Anne Vandenhende, ${ }^{1}$ Philippe Morlat, \\ Marie-Sylvie Doutre, ${ }^{2}$ Fabrice Bonnet ${ }^{1}$
}

To cite: Saunier A, Issa N, Vandenhende M-A, et al. Treatment of polyarteritis nodosa with tocilizumab: a new therapeutic approach?. RMD Open 2017;3:e000446. doi:10.1136/ rmdopen-2017-000446

- Prepublication history for this paper is available online. To view, these files please visit the journal online (http://dx.doi. org/10.1136/rmdopen-2017000446).

Received 14 February 2017 Revised 8 May 2017 Accepted 16 May 2017

\section{CrossMark}

${ }^{1}$ Service de Médecine Interne et Maladies Infectieuses, Hôpital Saint-André, CHU de Bordeaux, Bordeaux, France

${ }^{2}$ Service de Dermatologie, Hôpital Saint-André, CHU de Bordeaux, Bordeaux, France

Correspondence to Dr Fabrice Bonnet; fabrice. bonnet@chu-bordeaux.fr

\section{ABSTRACT}

We describe the effect of interleukin 6 (IL-6) blockade using tocilizumab (TCZ) for inducing and maintaining remission of refractory polyarteritis nodosa (PAN). Three patients with refractory PAN defined according to the American College of Rheumatology criteria were treated with TCZ infusions $(8 \mathrm{mg} / \mathrm{kg})$ on a monthly basis. All of them had severe cutaneous and articular involvement with elevated biological inflammatory markers. One suffered from a neuritis multiplex and one from renal and digestive damage. All three patients were dependent on high doses of glucocorticoids (above $0.5 \mathrm{mg} / \mathrm{kg}$ ) and two of them were resistant to immunosuppressive drugs. All patients achieved and maintained clinical response and normalisation of the inflammation acute-phase proteins after a few weeks of treatment with TCZ. Prednisolone could be reduced by an average of $41-13 \mathrm{mg} /$ day. These first case reports suggest that IL-6 blockade using TCZ could be a therapeutic alternative to induce remission in patients with polyarteritis nodosa resistant or intolerant to the reference treatment.

Polyarteritis nodosa (PAN) is a systemic necrotising vasculitis of the medium-sized and small-sized arteries. It has been associated with hepatitis B virus (HBV) infection in around $36 \%$ of cases and has become less common due to the discovery and widespread use of antiviral agents against HBV, HBV vaccines and the improved safety of blood transfusion. The annual incidence of PAN currently ranges from 0 to 1.6 cases per million inhabitants in Europe. ${ }^{1}$ The clinical presentation is most commonly associated with constitutional symptoms (eg, fever, weight loss, myalgia and arthralgia), and the disease spectrum ranges from involving one organ to polyvisceral failure. ${ }^{2}$ The thrombosis or rupture of the inflamed arteries induces ischaemia or haemorrhage in different organs with a risk of life-threatening complications. There is currently no biological test to facilitate diagnosis. Diagnosis is therefore primarily based on the results of histopathological samples

\section{Key messages}

What is already known about this subject?

- Corticosteroids and cyclophosphamide are the recomended treatments for polyarteritis nodosa (PAN) but they are not always effective or well tolerated.

What does this study add?

- Three patients with PAN had a dramatic therapeutic response to tocilizumab.

How might this impact on clinical practice?

- Tocilizumab could be a promising therapeutic appoach for patients with PAN.

showing vascular inflammatory lesions mixed with lymphocytes, macrophages, neutrophils, eosinophils with frequent fibrinoid necrosis.

Treatment of primary PAN is currently based on glucocorticoids (GC) and cyclophosphamide (CYC) and is guided by the Five-Factor Score. ${ }^{3}$ The duration of treatment is at least 12 months, but relapses occur in between $20 \%$ and $50 \%$ of HBV-negative patients after 2 years. ${ }^{4}$ These traditional treatments are not always effective or well tolerated in the long run and new anti-inflammatory strategies required for these rare inflammatory diseases.

Here, we present three case reports of patients with primary PAN, according to the American College of Rheumatology criteria, successfully treated with tocilizumab, an anti-interleukin 6 (IL-6) therapy.

\section{CASE REPORTS}

\section{Case 1}

A 39-year-old woman was in care since 2006 for systemic PAN revealed by a necrotic purpura . The initial diagnosis was based on the presence of small and medium vessel vasculitis on the deep skin biopsy, an aneurysm on renal artery imaging, negative immunological tests 
(cryoglobulin, antiphospholipid, antineutrophil cytoplasmic, antinuclear antibodies, rheumatoid factor) and negative HBV serology (table 1). She was successively treated with methotrexate (MTX), then GC $(1 \mathrm{mg} / \mathrm{kg}$ / day) and mycophenolate mofetil (2 g per day), nine intravenous bolus of CYC relayed by oral CYC $(3 \mathrm{mg} / \mathrm{kg} /$ day) with partial efficacy on constitutional symptoms and biological inflammatory syndrome $(\mathrm{C}$ reactive protein (CRP) between 59 and $126 \mathrm{mg} / \mathrm{L}$ over the following months). CYC was replaced by infliximab $5 \mathrm{mg} / \mathrm{kg}$ every 6 weeks associated with low-dose GC and MTX (10 mg per week) over a period of 4 years, but infliximab was stopped because of a paradoxical psoriasis due to antitumour necrosis alpha $(\mathrm{TNF} \alpha)$, resulting in a relapse of PAN. CYC pulse therapy and GC $(1 \mathrm{mg} / \mathrm{kg} /$ day $)$ were restarted, but the patient remained dependent on high levels of GC ( $>40 \mathrm{mg} /$ day). Intravenous TCZ $8 \mathrm{mg} / \mathrm{kg}$ was initiated in December 2015 and repeated every 4 weeks. The patient described a dramatic improvement of her general health status with a progressive decrease of CRP to $1 \mathrm{mg} / \mathrm{L}$ which allowed for tapering GC from $50 \mathrm{mg}$ to $10 \mathrm{mg} /$ day after 4 months of TCZ (figure 1A). One year later, she is still asymptomatic with no signs of inflammatory syndrome. She is still receiving TCZ every 4 weeks and $5 \mathrm{mg}$ of prednisolone per day.

\section{Case 2}

A 52-year-old woman in care since 2009 for a necrotic purpura and arthritis had a medical history involving obesity, diabetes and chronic obstructive pulmonary disease. Treatment in the early years was mainly based on colchicine with low-dose GCs and dapsone since 2010 (table 1).

In 2013, the patient's skin condition degraded abruptly. She experienced a wide range of skin necrosis, livedo, bubbles and intraoral ulcerations. The deep subcutaneous biopsy found a vasculitis of small and medium vessels. HBV serology was negative. MR angiography of the right arm showed multiple aneurysms. The patient received MTX associated with GC $(60 \mathrm{mg} /$ day $)$, which lacked efficacy. Extensive and destructive cutaneous and muscle lesions led to a more aggressive approach with pulse CYC and finally, oral CYC at $3 \mathrm{mg} / \mathrm{kg} /$ day with GC $(0.7 \mathrm{mg} / \mathrm{kg} /$ day $)$ followed by azathioprine and resulted in the remission of PAN. The haematological toxicity of immunosuppressive treatments hampered our ability to increase azathioprine to the target dose, and the patient relapsed as soon as GCs were decreased to below $25 \mathrm{mg}$ / day. The patient was disabled by anaemia $(8 \mathrm{~g} / \mathrm{dL})$ and osteoporosis with vertebral fractures.

As sparing corticosteroids and immunosuppressive drugs became crucial, TCZ $8 \mathrm{mg} / \mathrm{kg}$ intravenous was introduced and resulted in the clinical remission of PAN and the tapering of corticosteroids from 35 to $9 \mathrm{mg}$ over 6 months (figure 1b). At 1 year of treatment, she remains asymptomatic receiving TCZ every 4 weeks and $5 \mathrm{mg}$ of prednisolone per day.

\section{Case 3}

A 35-year-old man with no significant prior medical history presented suffering from multiple arthritis, myalgia, tenosynovitis, subcutaneous nodules, paraesthesia in the dominant hand, weight loss and biological inflammatory syndrome was referred to our institution (table 1). HBV serology and all the immunological tests were negative. CRP was elevated at $393 \mathrm{mg} / \mathrm{L}$. He was initially successfully treated with prednisolone $(80 \mathrm{mg} /$ day) but reported a relapse when prednisolone was reduced to around $40 \mathrm{mg} /$ day. 18 F-fluorodeoxyglucose positron emission combined with CT (FDG-PET/CT) revealed increased FDG uptake in the bone marrow and multiple subcutaneous and intramuscular nodules (maximum standardised uptake value at 5.6) (figure 2). A deep skin biopsy with necrosis inflammation of small-sized and medium-sized vessels led to the diagnosis of PAN.

The patient was treated by oral prednisolone $(1 \mathrm{mg} /$ $\mathrm{kg} /$ day) and intravenous immunoglobulin $(2 \mathrm{~g} / \mathrm{kg})$ with a modest and a short-term (less than 1 week) effect on CRP and poor clinical benefit. Given the high levels of inflammation, general symptoms and the dependence of very high doses of GC, TCZ $8 \mathrm{mg} / \mathrm{kg}$ was introduced. Three days after the start of the treatment, the patient reported an improvement of his general health status with a decreasing CRP to $12 \mathrm{mg} / \mathrm{L}$. The condition continued to improve despite the reduction of prednisolone from 60 to $30 \mathrm{mg}$ over the following 3 months (figure 1c). The FDG-PET/CT, conducted 4 months after the beginning of TCZ, was normal (figure 2). Ten months after starting treatment, the patient no longer requires corticosteroids. We have, therefore, begun to space out the TCZ infusion to every 6 weeks.

\section{DISCUSSION}

While the physiopathology of idiopathic systemic PAN is less well-known, the fundamental role of proinflammatory cytokines is no longer questioned. General symptoms and elevation of CRP are frequent and significant in PAN. IL-6 levels are very elevated in large vessels vasculitis and associated with constitutional symptoms and CRP. The essential function of TNF alpha in the expression of major histocompatibility complex class II suggests a central role of $\mathrm{T}$ cells in this pathology, major macrophage activation and a release of proinflammatory cytokines such as IL-1 and IL-6. ${ }^{2}$

TCZ is now a new therapeutic option for large vessel vasculitis (giant-cell arteritis or Takayasu disease) for patients resistant to or dependent on GCs. ${ }^{5-7}$ One case of amyloidosis secondary to PAN treated with tocilizumab, previously reported, also showed a good response of inflammatory markers including serum amyloid A protein. ${ }^{8}$ The role of proinflammatory IL- 6 , the data observed in patients with large vessel vasculitis and this other case of PAN treated by TCZ, argue the use of tocilizumab in our three patients suffering for refractory PAN. All patients have consented to receive TCZ and are still under treatment at this time. 


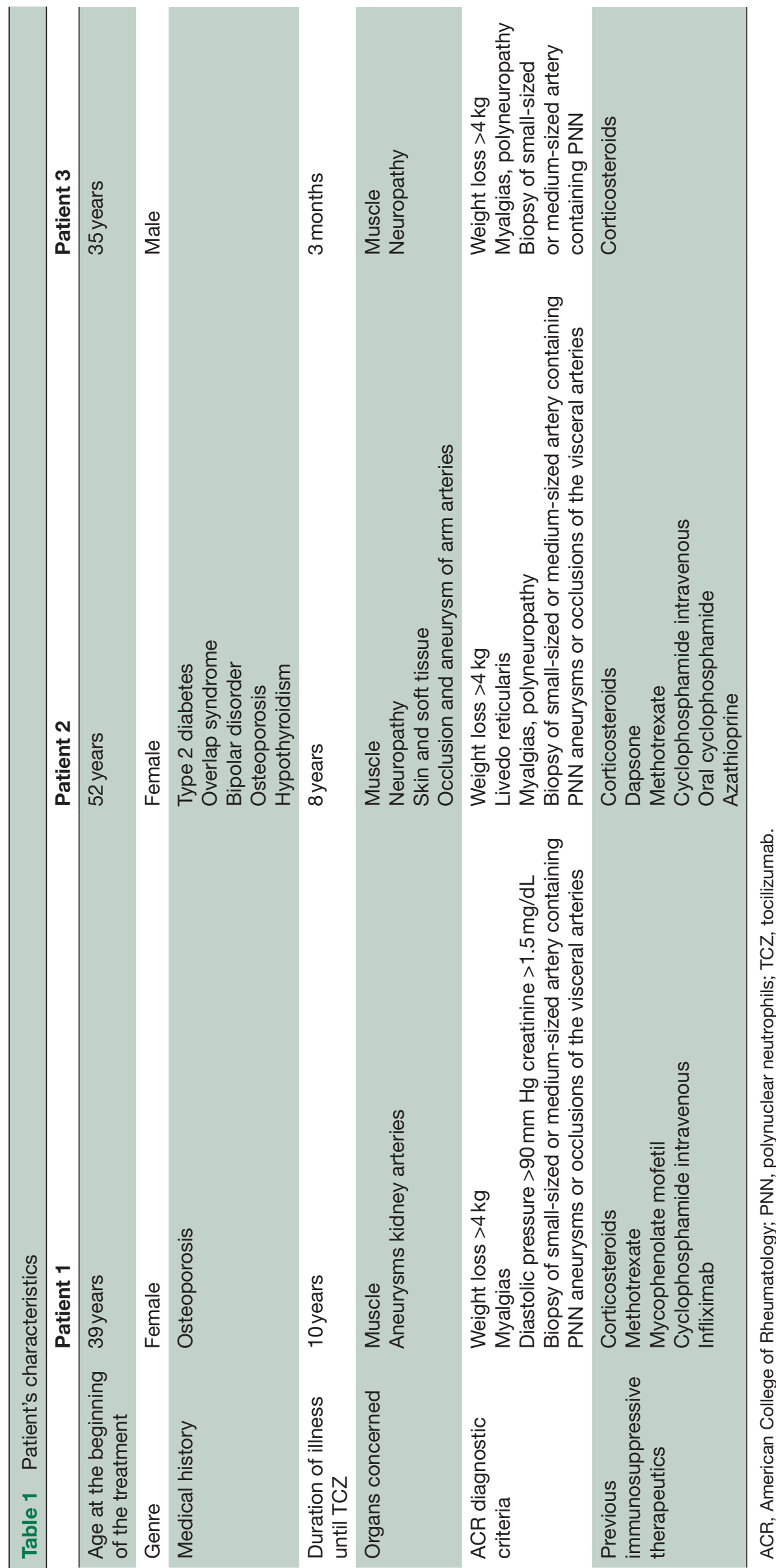




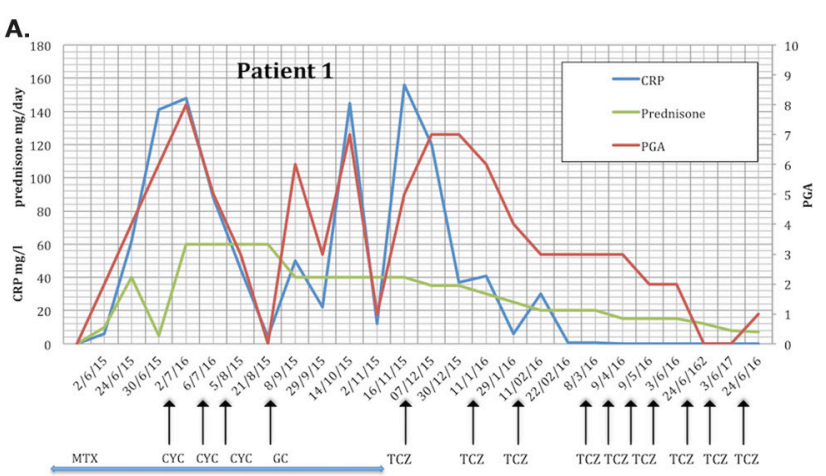

B.
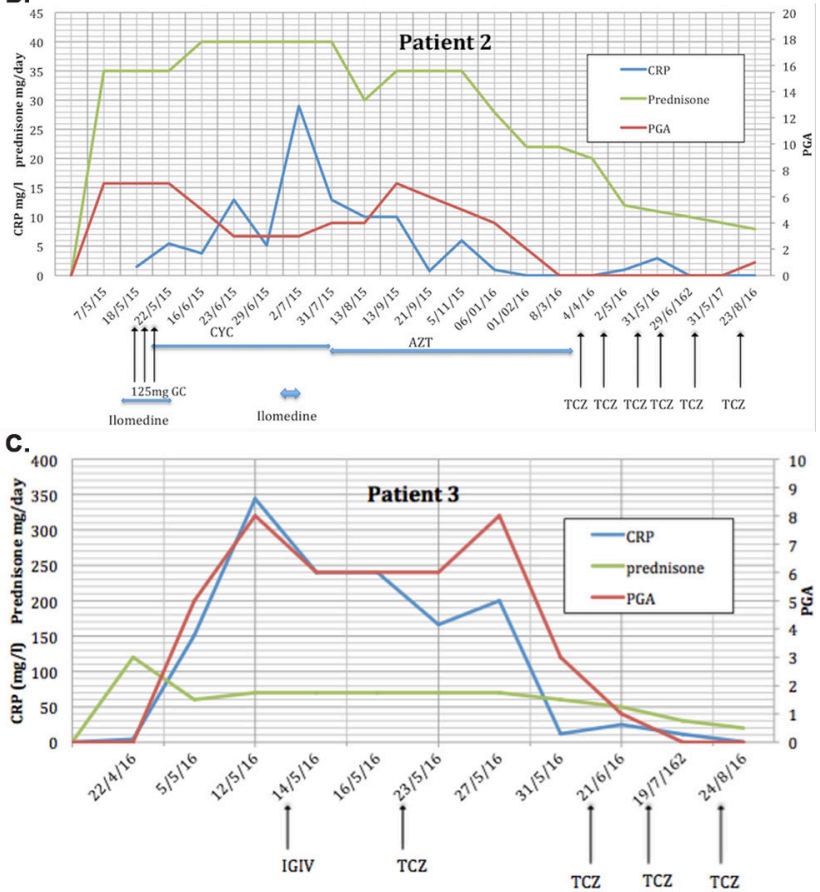

Figure 1 Patients' markers of disease activity and treatment. CRP (mg/L), dose of GC ( $\mathrm{mg}$ per day) and PGA (0 to 10). AZT, azathioprine; CRP, C reactive protein; CYC, cyclophosphamide; GC, glucocorticoids; IGIV, intravenous immunoglobulin; MTX, methotrexate; PGA, physician global assessment; TCZ, tocilizumab.

If TCZ is known to artificially decrease CRP levels, all of our patients presented dramatic clinical improvement which allowed us to significantly reduce the doses of GCs. The tolerance of TCZ in our patients and generally in patients with other indications remains good..$^{5-7}$

A few patients with PAN have been treated with other biological agents such as anti-TNF agents infliximab and etanercept which, in the reported cases, ${ }^{9}{ }^{10}$ has generally resulted in a good clinical response. However, vasculitis has also been described as a complication of anti-TNF agent infliximab and etanercept, making the choice of this agent difficult in the context of PAN. ${ }^{11}$ There are no data on the efficacy of rituximab in PAN. Thus, IL-6 may be the right target for PAN treatment like it has been recently shown for giant-cell arteritis. ${ }^{5}$

Many uncertainties remain regarding duration of treatment, long-term side effects in this context and its place

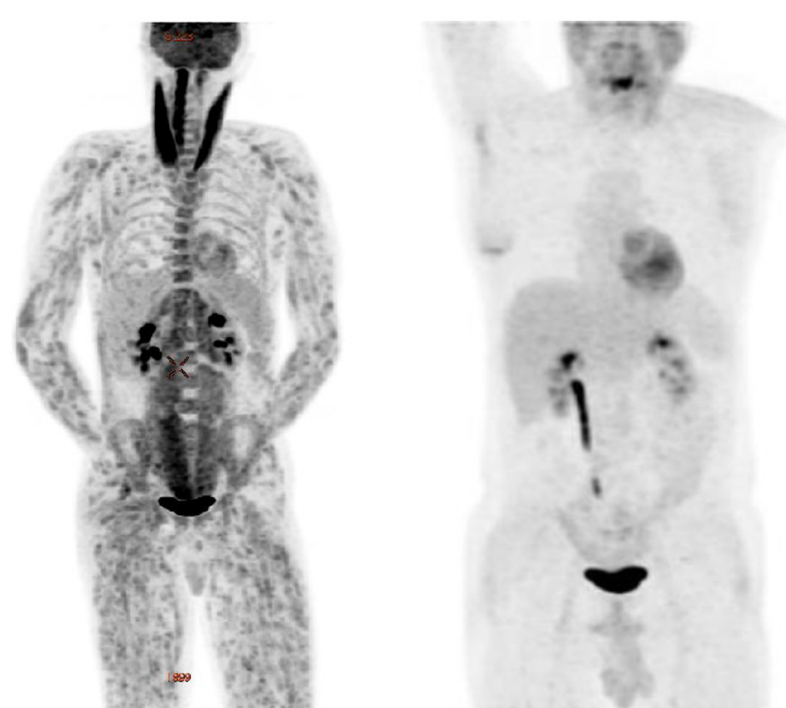

Figure 2 FDG-PET/CT of the third patient before treatment and 4 months later. FDG-PET, 18 F-fluorodeoxyglucose positron emission tomography.

as first-line treatment, treatment for relapses or to spare GCs. However, regarding the low incidence of idiopathic PAN and the issue of therapeutic trials in rare diseases, it is inconceivable to wait for results of controlled trials to allow patients with PAN to benefit from the TCZ in case of refractory PAN or more frequently to prevent GC long-term side effects in a pathology with a very limited therapeutic arsenal.

Acknowledgements The authors thank Diana Barger for the English revision of the manuscript.

Contributors All authors have participated to the management of these patients, to the collection of the data and to the analyses of the results. All authors have approved the manuscript before submission.

Competing interests None declared.

Provenance and peer review Not commissioned; externally peer reviewed. Data sharing statement Data can be obtained from the corresponding author. Open Access This is an Open Access article distributed in accordance with the Creative Commons Attribution Non Commercial (CC BY-NC 4.0) license, which permits others to distribute, remix, adapt, build upon this work non-commercially, and license their derivative works on different terms, provided the original work is properly cited and the use is non-commercial. See: http://creativecommons.org/ licenses/by-nc/4.0/

(C) Article author(s) (or their employer(s) unless otherwise stated in the text of the article) 2017. All rights reserved. No commercial use is permitted unless otherwise expressly granted.

\section{REFERENCES}

1. Hernández-Rodríguez J, Alba MA, Prieto-González S, et al. Diagnosis and classification of polyarteritis nodosa. J Autoimmun 2014:48-49:84-9.

2. De Virgilio A, Greco A, Magliulo G, et al. Polyarteritis nodosa: a contemporary overview. Autoimmun Rev 2016;15:564-70.

3. Guillevin L, Cohen P, Mahr A, et al. Treatment of polyarteritis nodosa and microscopic polyangiitis with poor prognosis factors: a prospective trial comparing glucocorticoids and six or twelve cyclophosphamide pulses in sixty-five patients. Arthritis Rheum 2003;49:93-100.

4. Samson M, Puéchal X, Devilliers H, et al. Long-term follow-up of a randomized trial on 118 patients with polyarteritis nodosa or microscopic polyangiitis without poor-prognosis factors. Autoimmun Rev 2014;13:197-205. 
5. Villiger PM, Adler S, Kuchen S, et al. Tocilizumab for induction and maintenance of remission in giant cell arteritis: a phase 2, randomised, double-blind, placebo-controlled trial. Lancet 2016;387:1921-7.

6. Nakaoka Y, Higuchi K, Arita Y, et al. Tocilizumab for the treatment of patients with refractory takayasu arteritis. Int Heart $J$ 2013:54:405-11.

7. Mekinian A, Comarmond C, Resche-Rigon M, et al. Efficacy of biological-targeted treatments in Takayasu arteritis: multicenter, retrospective study of 49 patients. Circulation 2015;132:1693-700.
8. Hočevar A, Lestan B, Šemrl SS, et al. AA amyloidosis in a polyarteritis nodosa patient treated with tocilizumab. Amyloid 2013;20:275-6.

9. Al-Bishri J, le Riche N, Pope JE. Refractory polyarteritis nodosa successfully treated with infliximab.J Rheumatol 2005;32:1371-3.

10. Feinstein J, Arroyo R. Successful treatment of childhood onset refractory polyarteritis nodosa with tumor necrosis factor alpha blockade. J Clin Rheumatol 2005;11:219-22.

11. Mohan N, Edwards ET, Cupps TR, et al. Leukocytoclastic vasculitis associated with tumor necrosis factor-alpha blocking agents. $J$ Rheumatol 2004;31:1955-8. 